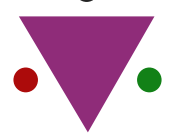

IJCRR

Section: Healthcare

Sci. Journal Impact

Factor: $6.1(2018)$

ICV: 90.90 (2018)

(c) (1) (9)

Copyright@IJCRR

\title{
Maxillary and Mandibular Dental Arch Dimensions in Saudi Population
}

\section{Mohammad Aljanakh ${ }^{1}$, Pavan Kumar Koralakunte Ravi²}

'Associate Professor, Department of Restorative Dentistry, College of Dentistry, University of Ha'il, Saudi Arabia; 'Professor, Department of Prosthodontics, Bapuji Dental College and Hospital, Davangere, Karnataka, India.

\section{ABSTRACT}

Rationale: Dental arch dimensions are not static; they change systematically during the period of intensive growth and development and less so in adulthood.

Objectives: To assess maxillary and mandibular dental arch dimensions of the Saudi population. Methods: Alginate impressions were made of the maxillary and mandibular dental arches of 100 male and female subjects aged between $20-28$ years to obtain study casts. The dental cast analysis was done to measure arch widths, arch lengths and palatal depths using Boley's guage by a standardized method. The measurements of maxillary and mandibular arch dimensions were tabulated and analyzed using independent test and Pearson's correlation test.

Results: Male gender showed increased arch dimensions in both maxillary and mandibular arches as compared to females. The difference was found to be statistically significant except for maxillary anterior and molar arch lengths. A strong positive correlation $(r=0.8, p<0.05)$ was observed between maxillary inter canine width and total arch length among females. Conclusion: The determined maxillary and mandibular dental arch dimensions are useful in selecting accurate stock impression tray, selection and arrangement of artificial teeth among Saudi edentulous patients undergoing prosthetic rehabilitation.

Key Words: Maxillary arch dimension, Mandibular arch dimension, Arch width, Arch length, Palatal depth

\section{INTRODUCTION}

The achievement of dental esthetics is one of the prime objectives of prosthodontic treatment for dentulous and edentulous patients. The key factor in achieving this goal is the determination of arch form, arch width, arch length and palatal contour. ${ }^{1}$ Dental arch dimensions are not static; they change systematically during the period of intensive growth and development and less so in adulthood. The values of the dimensions of the arch include its width, depth and circumference, inter canine and intermolar distances, overjet and overbite, which change during growth in different ways (the width of the teeth remains the same, whereas the lengths of the mandibular and maxillary bones increase). Also, the dental arch length varies with different races and gender. ${ }^{2}$

The palatal vault is defined as the deepest and most superior part of the palate or the curvature of the palate. ${ }^{3}$ The form of the palatal vault gives an idea to the original form of the dental arch before removal of the natural teeth and resorption of the residual ridge. ${ }^{4}$ The knowledge of arch size in prosthetic dentistry will help in the selection of correctly shaped stock impression trays, the size of artificial teeth and the overall form of the artificial dental arch at the wax trial stage that is amenable for modification by the prosthodontist. $^{5}$

Recent studies are done on the determination of arch dimensions and arch forms in both males and females subjects of Saudi Arabian population which are related mainly towards orthodontic treatment aspects but very few project concerning prosthodontic treatment. ${ }^{6,7,8}$ Hence, the present research study was conducted to determine the palatal depths, the maxillary and mandibular arch widths and the arch lengths among the male and female group. The results obtained from this study would facilitate in the accurate selection of stock impression trays and arrangement of the artificial teeth in an ideal dental arch form and dimensions that are closely

\section{Corresponding Author:}

Dr. Pavan Kumar Koralakunte Ravi, Department of Prosthodontics, Bapuji Dental College and Hospital, Davangere-577004, Karnataka India; Mobile: +919738416552; E-mail: pavan_dent@yahoo.co.in

ISSN: 2231-2196 (Print)

Received: 12.07 .2020
ISSN: 0975-5241 (Online)

Revised: 03.09 .2020
Accepted: 16.10 .2020 
related to each male and female dentulous and edentulous patients of Saudi Arabian population.

\section{MATERIAL AND METHODS}

In the present study, a total of 100 dental students (50 males and 50 females) of Saudi origin aged between 20 to 28 years studying at College of Dentistry, University of Ha'il, Saudi Arabia were randomly selected. The purpose of this particular research study being conducted was explained to all students involved in the study in their language with a signed consent accepted by the research ethics committee having reference number: 55456/5/4.

\section{Inclusion and exclusion criteria}

The respondents were selected under the following criteria having complete dentition in good alignment with presence or absence of third molars, normal overjet and overbite with a proper molar and canine relationships. But the respondents were excluded from the study under the following criteria - having any restorations, extensive carious lesions, incisal/occlusal wear, tooth fractures, gingival and periodontal problems, infections, congenital or surgical facial defects or developmental abnormalities, who have undergone orthognathic surgery and orthodontic treatment and those with history of oral habits like thumb sucking or mouth breathing.

The maxillary and mandibular casts were obtained from Alginate impressions made using disposable dentulous impression trays poured in type III dental stone following thorough infection control protocol which was divided into three sagittal and three transverse parts representing the dental arch widths and the dental arch lengths (Figure 1 and Figure 2) measured using Vernier calliper or Boley's guage. ${ }^{9}$

\section{Arch width measurements}

Maxillary and the mandibular inter canine widths and $1^{\text {st }}$ and $2^{\text {nd }}$ molar widths measurements were taken as follows:

a) Inter canine width (ICW): the linear distance between the cusp tip of the right and left canines.

b) Inter first molar width (1 $\left.1^{\text {st }} \mathrm{IMW}\right)$ : the linear distance between the mesiobuccal cusp tip of the right and left first molars.

c) Inter second molar width ( $2^{\text {nd }}$ IMW): the linear distance between the distobuccal cusp tip of the right and left second molars. ${ }^{910,11,12}$

\section{Arch length measurements}

Maxillary and mandibular anterior arch length, molar arch length and total arch lengths measurements were taken as follows:

a) Anterior arch length (AAL): the vertical distance from the midpoint of central incisors (incisal point) to the midpoint of the linear distance between the cusp tip of the right and left canines.

b) Molar arch length (MAL): the vertical distance from the midpoint of central incisors (incisal point) to the midpoint of the linear distance between the mesiobuccal cusp tip of the right and left first molars.

c) Total arch length (TAL): the vertical distance from the midpoint of central incisors (incisal point) to the midpoint of the linear distance between the distobuccal cusp tip of the right and left second molars. ${ }^{9,10,11,12}$

\section{Palatal depth measurements}

Palatal depth is the vertical distance from a point on the palatal width line to the palatal vault in the midline. The palatal depth is also represented by vertical distance from the midpoint of the linear distance between the palatal cusps of the two selected posterior teeth to the deepest point of the mid palatal suture measured by the vertical arm of the Vernier caliper or boley's gauge which includes any one of the following linear measurements as follows (Figure 3):

a) Inter first premolar depth: the linear distance between the palatal cusp tip of the right and left first premolars to the deepest point of the mid palatal suture.

b) Inter second premolar depth: the linear distance between the palatal cusp tip of the right and left second premolars to the deepest point of the mid palatal suture.

c) Inter first molar depth: the linear distance between the mesio palatal cusp tip of the right and left first molars to the deepest point of the mid palatal suture.

d) Inter second molar depth: the linear distance between the mesio palatal cusp tip of the right and left second molars to the deepest point of the mid palatal suture. ${ }^{9,10,13,14}$

The final values of the maxillary and mandibular arch widths (inter canine, inter $1^{\text {st }}$ and inter $2^{\text {nd }}$ molar widths), arch lengths (anterior, intermolar and total arch lengths) and the measurement of the palatal depth were tabulated.

\section{Statistical analysis}

Descriptive statistics were obtained by calculating mean, range, standard deviation using SPSS software (Version 11; SPSS inc, Chicago, IL, USA). Student's' test was done to compare the arch dimensions of males and females. Pearson's correlation coefficient was computed for arch lengths and arch widths. The significant level was fixed at $\mathrm{p}<0.05$.

\section{RESULTS}

Table 1 illustrates the comparison of mean maxillary arch dimensions between male and female groups. It was found that males had larger mean values of inter canine width (ICW), inter first molar width (IIMW), inter second molar 
width (I2MW), anterior arch length(AAL) palatal depth and molar arch length (MAL), when compared to females for the maxillary arch. The differences were found to be statistically significant with $\mathrm{p}<0.05$.

Table 2 illustrates the mean mandibular arch dimensions between male and female groups. It was found that overall males exhibited larger mean value dimensions when compared to females for the mandibular arch. The differences were found to be statistically significant with $p<0.05$.

Table 3 shows the correlation between arch widths and arch lengths. A strong linear relationship $(\mathrm{r}=0.8)$ was observed between maxillary total arch length and inter canine width among the female group. A statistically significant negative correlation was observed between maxillary molar arch lengths, inter canine width and $1^{\text {st }}$ molar width. But the $r$ value was $<0.5$. A moderate positive correlation was observed between maxillary total arch lengths and intermolar widths. The correlation was found to be statistically significant with $r<0.5$. The mandibular total arch length and arch width showed a statistically significant correlation.

\section{DISCUSSION}

A detailed extraoral and intraoral examination should be conducted in planning an individual treatment for each dental patient. We usually study and assess dental casts of patients requiring dental treatment in day to day clinical practice to provide oral rehabilitation and prosthodontic treatments. ${ }^{5,14}$

Gender-related differences in teeth, arch dimensions and palatal dimensions are statically different between sex, race and ethnic groups which are of great value to anthropologists and practising dentists. $2,4,9$ The knowledge about standards for teeth and arch dimensions for different races and ethnic groups would help in the choice of selecting stock impression trays, correct mould selection and setting of artificial teeth for fixed and removable dental prostheses to achieve optimal occlusion. ${ }^{5,10,12}$ Any alteration or change in the shape and position of the artificial teeth arrangement away from the natural arches will affect facial form and facial expression with inadequate esthetics and function of the dental prosthesis. ${ }^{13}$ The shape and the size of the artificial teeth selected should not exhibit extreme characteristics but need to harmonize with the face and profile of the patient..$^{15}$ This results in disappointment if the selection, arrangement and expectations between the patient and dentist do not match together. ${ }^{16}$

The overall mean values of the arch dimensions of the maxillary dental arch were larger than the mandibular dental arch both in widths and lengths in transverse and sagittal directions. ${ }^{13}$ This may be due to large teeth size in males compared to females which require wider arch for prosthetic teeth arrangement. The arch width was more than the arch length and arch width increased in the posterior segments due to square or ovoid arch forms in males and females. ${ }^{8}$ The gender differences in the dental arch dimensions in the present study can be related to the fact that the bony ridge thickness and alveolar process varies in both genders in that it was smaller in females than in males. Even the general muscular weakness plays a vital role in the facial width, breadth and length measurements of the dental arch in females and later growth in males compared to females. ${ }^{10,12,13}$ The physical characteristics and strong musculature in males than in females too play important role in facial development especially concerning the width and height of the maxillary dental $\operatorname{arch}^{5}$

It was found in the present study that the maxillary and mandibular dental arch dimensions in males were generally larger than females which were in line with previous studies. ${ }^{10,13,17}$ This may be due to the reason that teeth of Saudi males are larger than females and thus need a wider arch which is in line with the previous study. ${ }^{17}$ The largest difference existed in the inter second molar width distance may be due to differences in the dental arch forms between males and females in the sample group studied which is in line with the previous study. ${ }^{9}$ The maxillary molar arch length and total arch length in both males and females were almost similar and in line with the previous study. ${ }^{10}$

Different dental arch forms relate to inter canine width distances. The forms of dental arches are determined by measurements of inter canine width and anterior arch length. The results obtained in the present study are not in line with previous studies which may be because the pattern of craniofacial growth and development concerning different environmental, genetic and physical factors varies for a different population. ${ }^{5,11,12}$ In the present study, mean inter canine width and mean intermolar width in both arches were significantly higher in males than in females that are in line with the previous study which may be due to fact that size of the basal bone that is generally larger in males than in females. ${ }^{18}$ In the previous study, it was found no significant difference between mean mandibular inter canine width in both genders which is in disagreement with the present study. ${ }^{18}$

A weaker correlation was found between all the maxillary dental arch widths and lengths in males but moderate to stronger correlation was seen in females except between maxillary inter second molar width and total arch length. Different relationships between maxillary and mandibular dental arch dimensions represented highly complex interaction between various genetic and environmental factors. ${ }^{9,12}$ The palatal depth is significantly higher in males than in females which reflect overall greater breadth or width of male skulls than females in the present Saudi population sample studied which is line with previous studies. ${ }^{4,10}$ 
The hard palate morphology is related to the facial growth pattern. The palatal depths low is generally seen in short face type, medium in medium face type and high in long face type respectively. In our study, we have failed to assess the relationship between various facial types and hard palate morphology.

\section{CONCLUSION}

This study helps in selecting the correct maxillary and mandibular impression stock trays that will generally accommodate in the Saudi population group, thus making the tray selection process easier. Also, helps with the correct selection of artificial denture teeth and teeth setting for partial and completely edentulous patients.

\section{ACKNOWLEDGEMENT}

Authors acknowledge the immense help received from the scholars whose articles are cited and included in references of this manuscript.

\section{Conflict of interest: Nil}

Financial support: Nil

\section{REFERENCES}

1. Sellen PN, Jagger DC, Harrison A. Computer-generated study of the correlation between tooth, face, arch forms, and palatal contour. J Prosthet Dent 1998;80(2):163-168.

2. Kareem FA, Rasheed TA, Rauf AM. Longitudinal changes in dental arch circumference in Sulaimani city. European Scientific Journal. 2013;9(18):109-119.

3. The glossary of prosthodontic terms. $9^{\text {th }}$ ed. J Prosthet Dent 2017;117(5S):e1-e105.
4. Ahmad ZM. Palatal dimensions and its correlation with the circumference of upper anterior teeth. Al-Rafidain Dent J 2009;9(2):259-267.

5. Hamdi AQ. Maxillary arch dimensions in an Iraqi population sample. Anb Med J 2013;11(1):79-84.

6. Omar H, Alhajrasi M, Felemban N, Hassan A. Dental arch dimensions, form and tooth size ratio among a Saudi sample. Saudi Med J 2018;39(1):86-91.

7. Alkadhi OH, Almahfouz SF, Tokhtah HA, Binhuwaishel LA. Dental arch dimensions in Saudi adults. Int J Dent 2018;1:1-10.

8. Mohammad A, Koralakunte PR. Gender identification and morphologic classification of the tooth, arch and palatal forms in Saudi population. J Pharm Bioallied Sci 2015;7:S486-490.

9. Al-Zubair NM. Establishment of Yemeni dental arch form. Orthod J Nepal 2013;3(2):22-26.

10. Al-Zubair NM. Determinant factors of Yemeni maxillary arch dimensions. Saudi Dent J 2015;27(1):50-54.

11. Al-Zubair NM. Determinants factors of Yemeni dental arch forms. J Orthod R 2014;2(2):55-59.

12. Al-Zubair NM. The relationship between mandibular arch length and widths in a sample of Yemeni subjects with the normal dento-Skeletal relationship. J Orthod Sci. 2013;2(4):120-123.

13. Ahmed ZM. Clinical measurements of the dimensions of the dental arches and its application on the construction of a dental prosthesis. Al-Rafidain Dent J 2006;6(1):88-97.

14. Ahmed N, Fida M. A comparison of arch dimensions among different malocclusion groups. J Pak Dent Assoc 2010;19(2):94-98.

15. Koralakunte PR, Budihal DH, Basavapura ND. A classification of face form and tooth form - An invivo study. Int J Innov Med Health Sci 2013;1:14-19.

16. Koralakunte PR, Budihal DH. A clinical study to evaluate the correlation between maxillary central incisor tooth form and face form in an Indian population. J Oral Sci. 2012;54:273-78.

17. Hassan AK, Najim ZN. Measurement of the mandibular dental arch in Baghdad patients. East Mediterr Health J 2000;6(56):993-996.

18. Daniel MJ, Khatri M, Srinivasan SV, Jinsha VK, Marak F. Comparison of inter canine and intermolar width as an aid in gender determination: A preliminary study. J Indian Acad Forensic Med. 2014;36(2):168-72.

Table 1: Maxillary Arch Dimensions According to Gender

\begin{tabular}{|c|c|c|c|c|}
\hline Arch Dimensions & Gender & No of Participants & Mean \pm SD $($ SEM $)$ & P value \\
\hline Maxillary Inter Canine Width (ICW) & $\begin{array}{l}\text { Male } \\
\text { Female }\end{array}$ & $\begin{array}{l}50 \\
50\end{array}$ & $\begin{array}{c}35.74 \pm 2.221(0.314) \\
34.08 \pm 2.266(0.321)\end{array}$ & $<0.05$ \\
\hline Maxillary Inter first molar width ( $1^{\text {st }}$ IMW) & $\begin{array}{l}\text { Male } \\
\text { Female }\end{array}$ & $\begin{array}{l}50 \\
50\end{array}$ & $\begin{array}{c}51.00 \pm 2.483(0.351) \\
49.82 \pm 2.569(0.363)\end{array}$ & $<0.05$ \\
\hline Maxillary Inter Second molar width ( $2^{\text {nd }}$ IMW) & $\begin{array}{l}\text { Male } \\
\text { Female }\end{array}$ & $\begin{array}{l}50 \\
50\end{array}$ & $\begin{array}{l}58.52 \pm 4.082(0.577) \\
56.62 \pm 3.282(0.464)\end{array}$ & $<0.05$ \\
\hline Maxillary Anterior arch length (AAL) & $\begin{array}{l}\text { Male } \\
\text { Female }\end{array}$ & $\begin{array}{l}50 \\
50\end{array}$ & $\begin{array}{l}9.80 \pm 1.385(0.196) \\
9.36 \pm 1.120(0.158)\end{array}$ & $>0.05$ \\
\hline Maxillary Molar Arch Length (MAL) & $\begin{array}{l}\text { Male } \\
\text { Female }\end{array}$ & $\begin{array}{l}50 \\
50\end{array}$ & $\begin{array}{l}30.34 \pm 2.264(0.320) \\
29.64 \pm 1.860(0.263)\end{array}$ & $>0.05$ \\
\hline Maxillary Total Arch Length (TAL) & $\begin{array}{l}\text { Male } \\
\text { Female }\end{array}$ & $\begin{array}{l}50 \\
50\end{array}$ & $\begin{array}{c}46.98 \pm 2.263(0.320) \\
45.56 \pm 2.581(0.365)\end{array}$ & $<0.05$ \\
\hline Maxillary Palatal Depth & $\begin{array}{l}\text { Male } \\
\text { Female }\end{array}$ & $\begin{array}{l}50 \\
50\end{array}$ & $\begin{array}{l}21.02 \pm 2.325(0.329) \\
20.14 \pm 1.773(0.251)\end{array}$ & $<0.05$ \\
\hline
\end{tabular}

Note: SD - Standard Deviation, SEM - Standard Error of Mean 
Table 2: Mandibular Arch Dimensions According to Gender

\begin{tabular}{|c|c|c|c|c|}
\hline Arch Dimensions & Gender & No of Participants & Mean \pm SD $($ SEM $)$ & P-value \\
\hline Mandibular Inter Canine Width (ICW) & $\begin{array}{l}\text { Male } \\
\text { Female }\end{array}$ & $\begin{array}{l}50 \\
50\end{array}$ & $\begin{array}{l}28.04 \pm 2.060(0.291) \\
27.00 \pm 2.020(0.286)\end{array}$ & $<0.05$ \\
\hline Mandibular Inter First Molar Width (1 $1^{\text {st }}$ IMW) & $\begin{array}{l}\text { Male } \\
\text { Female }\end{array}$ & $\begin{array}{l}50 \\
50\end{array}$ & $\begin{array}{l}46.12 \pm 2.782(0.393) \\
44.02 \pm 2.412(0.341)\end{array}$ & $<0.05$ \\
\hline Mandibular Inter Second Molar Width ( $2^{\text {nd }}$ IMW) & $\begin{array}{l}\text { Male } \\
\text { Female }\end{array}$ & $\begin{array}{l}50 \\
50\end{array}$ & $\begin{array}{l}54.18 \pm 2.876(0.407) \\
51.06 \pm 2.766(0.391)\end{array}$ & $<0.05$ \\
\hline Mandibular Anterior Arch Length (AAL) & $\begin{array}{l}\text { Male } \\
\text { Female }\end{array}$ & $\begin{array}{l}50 \\
50\end{array}$ & $\begin{array}{c}6.02 \pm 1.204(0.170) \\
5.42 \pm 0.702(0.099)\end{array}$ & $<0.05$ \\
\hline Mandibular Molar Arch Length (MAL) & $\begin{array}{l}\text { Male } \\
\text { Female }\end{array}$ & $\begin{array}{l}50 \\
50\end{array}$ & $\begin{array}{l}25.60 \pm 1.604(0.227) \\
24.88 \pm 1.480(0.209)\end{array}$ & $<0.05$ \\
\hline Mandibular Total Arch Length (TAL) & $\begin{array}{l}\text { Male } \\
\text { Female }\end{array}$ & $\begin{array}{l}50 \\
50\end{array}$ & $\begin{array}{l}42.92 \pm 2.165(0.306) \\
40.80 \pm 2.563(0.363)\end{array}$ & $<0.05$ \\
\hline
\end{tabular}

Note: SD - Standard Deviation, SEM - Standard Error of Mean

Table 3: Relationship between maxillary and mandibular arch width and arch lengths according to gender

\begin{tabular}{|c|c|c|c|c|c|c|}
\hline \multirow[t]{2}{*}{ Maxillary Arch } & \multicolumn{2}{|c|}{ Anterior Arch Length } & \multicolumn{2}{|c|}{ Molar Arch Length } & \multicolumn{2}{|c|}{ Total Arch Length } \\
\hline & Male & Female & Male & Female & Male & Female \\
\hline Inter Canine Width & -0.064 & 0.077 & -0.278 & $-0.293^{*}$ & -0.042 & $0.805^{*}$ \\
\hline Inter $1^{\text {st }}$ Molar Width & -0.160 & -0.112 & -0.269 & $-0.313^{*}$ & -0.040 & $0.468^{\prime}$ \\
\hline Inter $2^{\text {nd }}$ Molar Width & -0.151 & -0.112 & -0.126 & -0.264 & -0.189 & $0.327^{*}$ \\
\hline \multicolumn{7}{|l|}{ Mandibular Arch } \\
\hline Inter Canine Width & -0.165 & 0.144 & -0.075 & -0.020 & $0.326^{*}$ & $0.433^{*}$ \\
\hline Inter $1^{\text {st }}$ Molar Width & -0.013 & -0.029 & -0.094 & -0.165 & -0.103 & $0.516^{*}$ \\
\hline Inter $2^{\text {nd }}$ Molar Width & -0.272 & 0.102 & -0.157 & -0.008 & -0.066 & $0.281^{*}$ \\
\hline
\end{tabular}

Note: * $\mathrm{p}<0.05$

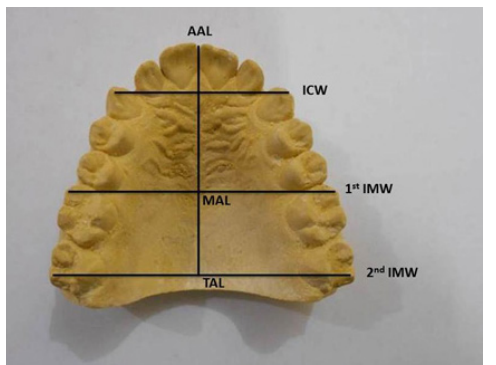

Figure 1: Maxillary arch dimensions showing widths (ICWInter canine width, $1^{\text {st }} 1 \mathrm{MW}$ - First inter molar width, $2^{\text {nd }}$ IMW - Second inter molar width) and lengths (AAL - Anterior arch length, MAL - Molar arch length, TAL - Total arch length).

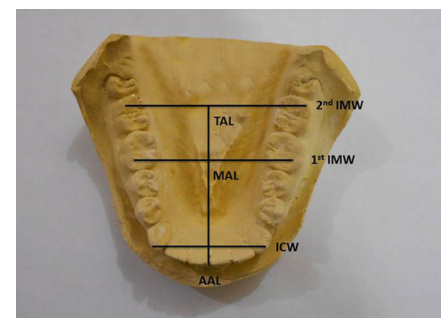

Figure 2: Mandibular arch dimensions showing widths (ICWInter canine width, $1^{\text {st }} 1 \mathrm{MW}$ - First inter molar width, $2^{\text {nd }} \mathrm{IMW}$ - Second inter molar width) and lengths (AAL - Anterior arch length, MAL - Molar arch length, TAL - Total arch length).

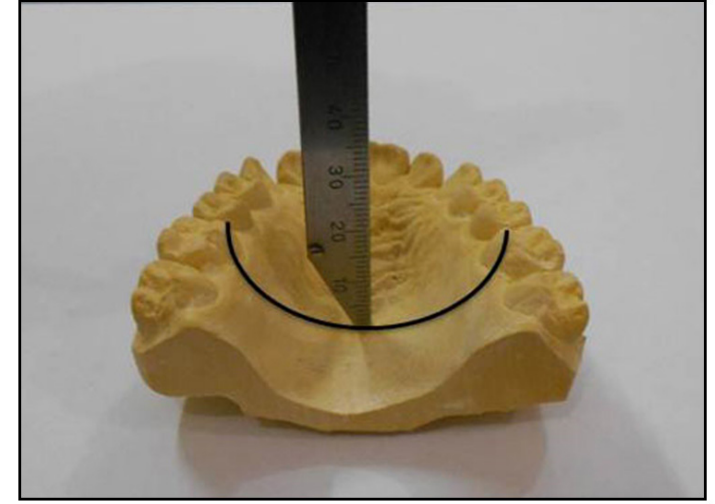

Figure 3: Palatal depth measurement at the deepest point using Boley's guage. 